\title{
Enfermagem e a implantação da Terapia Comunitária Integrativa na Estratégia Saúde da Família: relato de experiência
}

\author{
Nursing and the implementation of Integrative Community Therapy \\ in the Family Health Strategy: an experience report \\ Enfermería y la aplicación de la Terapia Comunitaria Integrativa en la Estrategia \\ de Salud de la Familia: un relato de experiencia
}

José Martins Jatai', Lucilane Maria Sales da Silva"

' Prefeitura Municipal de Fortaleza, Secretaria Municipal de Saúde, Programa de Saúde da Família. Fortaleza-CE, Brasil. " Universidade Estadual do Ceará, Curso de Graduação em Enfermagem, Programa de Pós-Graduação em Cuidados Clínicos em Saúde, Laboratório de Pesquisa e de Práticas Coletivas em Saúde (Coordenadora). Fortaleza-CE, Brasil.

\author{
Submissão: 07-10-2010 Aprovação: 16-08-2012
}

\section{RESUMO}

Terapia Comunitária Integrativa (TCl) é um instrumento de cuidado que nos permite tecer redes sociais e prevenir sofrimentos psíquicos. Com este artigo, objetivou-se relatar a experiência de implantação da TCl pelo enfermeiro na Estratégia Saúde da Família, vivenciada no período de setembro de 2008 a abril de 2009, na comunidade Alto Jerusalém, em Fortaleza, Ceará, Brasil, como parte do Curso de Formação de Terapeuta Comunitário Integrativo. O relato foi dividido em fases: sensibilização dos gestores e profissionais da saúde e estratégias de formação dos grupos. Concluímos que, como instrumento de cuidado, a TCI demonstrou atender os princípios norteadores do SUS, ensinando-nos a construir redes de apoio social, possibilitando mudanças e reconhecendo as competências de cada ator social para superação das dificuldades relatadas.

Descritores: Terapia; Comunidade; Estratégia Saúde da Família; Enfermagem.

\section{ABSTRACT}

Integrative Community Therapy (ICT) is an instrument of care that allows us to make social networks and prevent mental suffering. This paper aimed to report an experience of implementation of ICT by nurse in the Family Health Strategy, carried out from September 2008 to April 2009, at the Alto Jerusalem community, in Fortaleza, Ceará, Brazil, as part of a training course of Integrative Therapist Community. The report was divided into phases: awareness of managers and health professionals and training strategies of the groups. We conclude that, as an instrument of care, ICT meets the guiding principles of SUS, teaching us to build social support networks, enabling changes and recognizing the competences of each social actor to overcome the difficulties reported.

Key words: Therapy; Community; Health Program Strategy; Nursing.

\section{RESUMEN}

Terapia Comunitaria Integrativa (TCI) es un instrumento de la atención que nos permite hacer redes sociales y evitar el sufrimiento mental. El objetivo de este artigo fue relatar la experiencia de aplicación de la TCl por enfermero en la Estrategia de Salud de la Familia, vivida, de septiembre de 2008 a abril de 2009, en la comunidad Alto Jerusalén, Fortaleza, Ceará, Brasil, como parte del curso de Formación del Terapeuta en Terapia Comunitaria Integrativa. El informe se divide en fases: sensibilización de los directores y profesionales de la salud y estrategias de formación de los grupos. Llegamos a la conclusión de que, como un instrumento de la atención, la TCI ha demostrado cumplir con los principios rectores del SUS, nos enseñando a construir redes de apoyo social, permitiendo cambios y reconociendo las competencias de cada actor social para superar las dificultades señaladas. Palabras clave: Terapia; La comunidad; Estrategia de Salud Familiar; Enfermería.

\section{AUTOR CORRESPONDENTEＪosé Martins JataiＥ-mail: zuir@uol.com.br}




\section{INTRODUÇÃO}

O setor saúde, no Brasil, tem avançado no campo das políticas sociais, por adotar o Sistema Único de Saúde com seus princípios de Universalidade, integralidade, Equidade, pela descentralização e participação da comunidade ${ }^{(1)}$. A adoção desses princípios possibilitou a introdução de práticas que possam romper com as formas tradicionais do atendimento em saúde.

No campo da saúde mental a Reforma Psiquiátrica promoveu uma ruptura com o modelo hospitalocêntrico, assegurando uma política de reabilitação e inclusão social por meio de serviços de saúde mental, não hospitalares. Seguindo as mesmas diretrizes, na Estratégia Saúde da Família (ESF) também devem ser desenvolvidas ações de prevenção à doença mental e ao sofrimento psíquico. Para isso, os profissionais que atuam nessas equipes de saúde, devem estar capacitados para promover a saúde, prevenir o adoecimento mental, identificando situações e fatores de risco que provocam esse adoecimento ${ }^{(2)}$.

A enfermagem exerce papel importante no processo de cuidar na Estratégia Saúde da Família. A função de assistir a comunidade e desempenhar atividades de promoção e educação em saúde, manutenção e recuperação da saúde, prevenção às doenças, tratamento e reabilitação têm facilitado aos enfermeiros grande autonomia, resultando numa significante ascensão social e política da profissão ${ }^{(3)}$.

O profissional de enfermagem atuando na Estratégia Saúde da Família tem possibilidade de utilizar diversas estratégias para o oferecimento do cuidado em saúde. Nesse contexto, a Terapia Comunitária Integrativa ( $\mathrm{TCl}$ ) vem se consolidando como uma nova tecnologia de cuidado em saúde mental, constituindo-se em instrumento valioso de intervenção psicossocial na saúde pública, a mesma não pretende substituir outros serviços de saúde, mas complementá-los, de modo a ampliar as ações preventivas e promocionais. A TCl funciona como primeira instância de atenção básica, porque acolhe, escuta e cuida dos sujeitos e de seus sofrimentos e, desse modo, possibilita direcionar melhor as demandas. Com isso, permite que só afluam para os níveis secundários de atendimento situações que, devido à sua complexidade, exigem a intervenção complementar do especialista ${ }^{(4)}$.

A TCl é caracterizada como um espaço de promoção de encontros, interpessoais e intercomunitários onde se procura partilhar experiências de vida e sabedoria de forma horizontal e circular, objetivando a valorização das histórias de vida dos participantes, a restauração da auto-estima e da confiança em si, a ampliação da percepção dos problemas e possibilidades de resolução a partir das competências locais. Tem como base de sustentação o estímulo para construção de vínculos solidários e a promoção da vida. É uma prática de efeito terapêutico, destinada à prevenção na área da saúde e ao atendimento de grupos heterogêneos através do contato face-a-face, promovendo a construção de vínculos solidários por meio de uma rede de apoio social, onde a comunidade busca resolver os problemas de forma coletiva ${ }^{(4)}$.

O trabalho realizado pelos profissionais de saúde envolvidos na promoção da saúde mental baseia-se na proposta de um ambiente de escuta e acolhimento, a fim de estimular a mediação para prevenção e inserção social, proporcionando a formação de vínculos entre as pessoas e a partilha de experiências. Dessa forma, o terapeuta comunitário pode compreender melhor os problemas da comunidade e direcionar suas condutas terapêuticas, melhorando a qualidade de vida da população e economizando custos para os gestores ${ }^{(5)}$.

A prática da Terapia Comunitária Integrativa integra, desde 2008, a Política Nacional de Práticas Integrativas e Complementares (PNPIC), do Departamento de Atenção Básica, do Ministério da Saúde. Tem se revelado para os gestores de saúde e comunidade um instrumento de grande valor estratégico, uma preciosidade rumo à efetivação do Sistema Único de Saúde (SUS), respondendo dentro deste universo a importantes diretrizes como a equidade e universalidade, grandes fontes de inclusão e cidadania(6-7).

A prática da Terapia Comunitária Integrativa surgiu em 1986, na comunidade do Pirambú, uma das maiores favelas de Fortaleza-CE, Brasil, como resposta a uma crescente demanda de indivíduos com sofrimento psíquico que buscavam apoio jurídico junto ao Projeto de Apoio aos Direitos Humanos da favela. É uma construção metodológica do psiquiatra, antropólogo e professor universitário Dr. Adalberto de Paula Barreto, para atender às necessidades de saúde da população da referida comunidade. Atualmente, a TCl está implantada em alguns países como França e Suíça, com alguma experiência no Uruguai e Argentina aonde vem sendo desenvolvida por enfermeiras, além de estar presente em todos os estados brasileiros $^{(4,8)}$. No Brasil, atualmente, a Terapia Comunitária Integrativa está implantada em dezessete estados brasileiros. A partir das experiências nos vários cantos do Brasil, constata-se que o mais importante é transformar o unitário em comunitário, o individual em coletivo.

O objetivo deste estudo é relatar a experiência de implantação da prática da Terapia Comunitária Integrativa na Estratégia do Saúde da Família em uma unidade básica de saúde de um bairro periférico de Fortaleza, Ceará, Brasil. A opção por esta forma de apresentação deve-se ao fato de termos experimentado, atuando na ESF como profissionais de enfermagem, um espaço de diálogo que incluiu nossas emoções, nossas opiniões, os conceitos de autores, como também, as ideias e as falas daqueles que têm estado conosco nos últimos tempos refletindo e vivenciando o tema. Assim, esse trabalho foi construído em sintonia com a prática, com as emoções e com a pesquisa bibliográfica, além de incluirmos todas as "vozes" que reencontramos no caminho e que colaboraram para o significado que hoje damos para esse tema.

\section{EIXOS TEÓRICOS DA TERAPIA COMUNITÁRIA INTE- GRATIVA}

A Terapia Comunitária Integrativa tem construído sua identidade, alicerçada em cinco grandes pilares teóricos: Pensamento Sistêmico; Teoria da Comunicação; Antropologia Cultural, Pedagogia Freireana e Resiliência. Esses referenciais estão imbricados numa interpelação consistente e indissociável para a compreensão da metodologia da $\mathrm{TCl}$.

O Pensamento Sistêmico é uma maneira de abordar, de 
ver, de situar, de pensar um problema em relação ao seu contexto. Essa abordagem permite perceber a pessoa humana na sua relação com a família, a sociedade, com seus valores e crença, contribuindo, assim, para a compreensão e transformação do indivíduo ${ }^{(9-10)}$.

A Teoria da Comunicação proporciona inúmeras possibilidades de comunicação entre as pessoas, convidando-as a ir além das palavras para entender a busca desesperada de cada ser humano pela consciência de existir e pertencer, de ser confirmado e reconhecido, como indivíduo e cidadão, o que permite a ampliação de nossas próprias possibilidades de transformação e de resignificação ${ }^{(9-10)}$.

A Antropologia Cultural ressalta os valores culturais e as crenças como importante fator na formação da identidade do indivíduo e do grupo ${ }^{(9-10)}$.

A pedagogia Freireana nos lembra que ensinar não é apenas uma transferência de conhecimentos acumulados por um educador(a) experiente e que sabe tudo para um educando(a) inexperiente que não sabe nada. Ensinar é o exercício do diálogo, da troca, da reciprocidade, ou seja, de um tempo para escutar, de um tempo para aprender e de um tempo para ensinar ${ }^{(4)}$.

E, por último, a Resiliência, capacidade dos indivíduos, famílias e comunidades em superar as dificuldades contextuais, funciona como fonte importante do conhecimento que confere segurança, competência e saber ${ }^{(4,8-10)}$

\section{ETAPAS DE EXECUÇÃO DA TERAPIA COMUNITÁRIA}

A Roda de Terapia Comunitária Integrativa se desenvolve percorrendo as seguintes fases: 1- Acolhimento; 2- Escolha do tema; 3- Contextualização; 4- Problematização e, 5- Encerramento. No Acolhimento procura-se ambientar o grupo, colocando as pessoas bem à vontade e confortáveis, de preferência em círculo. Nesse momento o terapeuta comunitário apresenta uma síntese do que é a Terapia Comunitária e discorre sobre as regras ou condições para o funcionamento do grupo.

Na Escolha do Tema a fala fica aberta para os participantes apresentarem, de forma sucinta, os problemas ou situações que estão gerando preocupação. Após a exposição dos problemas, o grupo se manifesta escolhendo o tema daquela roda e dizendo sumariamente o porque da escolha.

Na fase de Contextualização é solicitado à pessoa cujo tema foi escolhido que explicite o problema ou situação apresentada. A pessoa em foco detalha a situação e, nesse momento, todos, inclusive os terapeutas, podem fazer perguntas para esclarecer melhor a questão. As perguntas vão ajudando na reordenação das idéias, quebra das certezas e, portanto na criação de disponibilidades para mudanças. Então o terapeuta condutor deve estar atento para extrair dos depoimentos da pessoa escolhida o mote - uma pergunta chave que vai permitir a reflexão do grupo durante a terapia. É um instrumento de transformação do problema a partir da dimensão individual para a dimensão grupal. Representa o ponto de encontro entre os participantes da terapia, aquilo que liga, solidariza, exprime a mesma humanidade dentro de cada um.

No momento em que o mote é retransmitido para o grupo chega-se na fase da Problematização. Nessa fase, o grupo se coloca atendendo à solicitação do mote, sempre falando de sua própria experiência. O grupo torna-se envolvido com o problema e as alternativas apresentadas passam a ser do próprio grupo. Entende-se que o grupo alcança então, uma compreensão diferenciada do problema assim como a própria pessoa que o expôs, pode vê-lo em diferentes vieses e compreensões.

No Encerramento, é proporcionado um ambiente de interiorização, de clima afetivo para que as pessoas se sintam apoiadas pelos outros. O terapeuta procura fazer conotações positivas a todos que se expuseram ou apresentaram seus sofrimentos e pede para que os participantes falem das coisas boas que mais lhes tocaram e admiraram. A sessão da $\mathrm{TCl}$ termina com os agradecimentos dos terapeutas e com convite para os próximos encontros.

\section{DESCRIÇÃO DA EXPERIÊNCIA}

A experiência relatada ocorreu no bairro Quintino Cunha, localizado na Zona Oeste da cidade de Fortaleza, área de cobertura da Estratégia Saúde da Família do Centro de Saúde George Benevides. Tudo começou a partir do curso de formação em terapeuta comunitário oferecido pelo Movimento Integrado de Saúde Mental do Ceará (MISMEC/CE). Diz um ditado popular, que: "só se aprende praticando", ou seja, tínhamos que transpirar TCl para que o nosso aprendizado realmente acontecesse. Então fomos à busca de grupos de pessoas da comunidade que iriam participar das terapias.

Mobilizar é convocar vontades para atuar na busca de um propósito comum, sob uma interpretação e um sentido também compartilhados. As pessoas são chamadas, mas participar ou não é uma decisão de cada uma. Esta decisão depende essencialmente das pessoas se verem ou não como responsáveis e como capazes de provocar e construir mudanças ${ }^{(11)}$. Mas como fazer? Por onde iniciar?

\section{O início - sensibilização dos gestores e profissionais de saúde}

A Terapia Comunitária Integrativa obedece a uma sequência já mencionada anteriormente que vai desde o acolhimento até o encerramento. Estávamos com a teoria e precisávamos unir com a prática.

O objetivo do curso era formar um enfermeiro e um agente comunitário de saúde da Estratégia Saúde da Família para realização de Terapias Comunitárias Integrativas junto às famílias assistidas pela equipe. O curso contava com 360 horas, entre parte teórica e prática. Era realizado através de encontros mensais no município de Beberibe, localizado na região leste do Estado. Ficávamos em regime de dedicação exclusiva, durante quatro dias seguidos, realizando práticas de Terapia Comunitária Integrativa entre os próprios alunos. Participávamos de encontros mensais chamados intervisões onde tirávamos dúvidas e trocávamos experiências. Esses encontros eram realizados no Projeto Quatro Varas no bairro do Pirambú, em Fortaleza-CE. A parte prática do curso era de, no mínimo, 48 terapias até o final da capacitação. Essa parte prática tinha que ser feita junto a nossa comunidade. 
Então como sensibilizar a coordenação local da nossa unidade e os demais profissionais de saúde? Esse processo de sensibilização ocorreu através de depoimentos dos agentes comunitários de saúde (ACS) que participaram da primeira terapia comunitária integrativa realizada dentro da própria unidade básica de saúde. E agora? Tínhamos que traçar estratégias de mobilização da comunidade para participarem da TCl.

\section{Estratégias de mobilização da comunidade para adesão ao grupo de $\mathrm{TCI}$}

Divulgação das Sessões de TCl: utilizamos, inicialmente, o convite informal durante as consultas dos profissionais de saúde, na visita do ACS e através de cartaz fixado dentro da unidade de saúde.

Horários: tínhamos que colocar em nosso cronograma de atendimento o dia e o horário de realizações da $\mathrm{TCl}$. O curso de formação exigia duas vezes na semana. Então colocamos uma sessão no período da manhã e a outra no período da tarde.

Local das terapias e formação dos grupos que iriam participar das terapias: a princípio o local ideal para as terapias seria na própria unidade de saúde. Entretanto, não tínhamos o espaço físico adequado. Então, buscamos espaços sociais dentro da própria comunidade. Dentre esses espaços estivemos no Centro Comunitário São Francisco, com grupos de terceira idade e gestantes; na Escola de Ensino Médio e Fundamental Jesus Maria José com grupos de estudantes; e com o grupo de moradores da comunidade Alto Jerusalém.

A comunidade do Alto Jerusalém está localizada no bairro do Quintino Cunha, região periférica de Fortaleza. Com aproximadamente 1.114 famílias, coberta por cinco agentes comunitários de saúde, estando situada em uma das 32 micro-áreas que fazem parte da área de adscrição do Centro de Saúde George Benevides - SER III, em Fortaleza-CE, sendo a comunidade geograficamente mais distante da unidade de saúde. Encontra-se próximo a uma região de mangue, sendo considerada uma área de risco 1 e 2 com baixos índices de desenvolvimento humano (desemprego; falta de saneamento básico e violência urbana). Existia no local uma associação comunitária que na prática não funcionava. Resolvemos então utilizar este espaço social para a utilização de nossas terapias. As pessoas eram convidadas a participar das terapias todas as quartas-feiras no período da tarde.

A quantidade de pessoas presentes, inicialmente, era pequena. A comunidade justificava não poder ir as terapias for falta de tempo, estavam dormindo ou não tinham interesse. Muitas vezes, pensamos em desistir e mudar de local. No entanto, decidimos mudar de estratégia, na tentativa de sensibilizar mais pessoas a participar. Foi então que surgiu a idéia de oferecer as terapias durante o acesso da população a outros serviços básicos de saúde, a princípio aos exames de Papanicolaou (prevenção do câncer de colo uterino). Como a comunidade era distante da Unidade de Saúde, e na maioria das vezes as muIheres não conseguiam marcar as consultas, ao conciliar a participação da terapia com o acesso às consultas pelas mulheres, a participação das mesmas foi considerável. E a ideia deu certo.

Daí por diante a divulgação ocorreu dentro da própria comunidade através das pessoas que participavam da Terapia Comunitária Integrativa.
A mobilização da comunidade em torno da participação de práticas diferenciadas, pode se dar de forma natural, mas também pode ser provocada por profissionais comprometidos com a qualidade de vida das pessoas, que incorporem em sua prática a socialização e discussão dos saberes que permeiam a área da saúde ${ }^{(12)}$.

\section{RESULTADOS E AVANÇOS DA EXPERIÊNCIA}

Atualmente as terapias acontecem na Associação Comunitária Alto Jerusalém, de forma sistemática, todas as quartas-feiras no período da tarde com uma média de 25 participantes, entre adultos, idosos e crianças. A condução da $\mathrm{TCl}$ é feita por um enfermeiro e um agente comunitário de saúde. Além dos participantes da própria comunidade, recebemos pacientes encaminhados da Unidade de Saúde da Família, do Centro de Atenção Psicossocial e Centro de Referência de Assistência Social. Antes do início das sessões da TCl, divulgamos atividades relacionadas à saúde coletiva, entre elas avisos sobre as campanhas de vacinação, a importância do pré-natal, da prevenção do câncer de colo uterino e mamas, combate a dengue, marcação de prevenção, marcação da avaliação da bolsa família, dentre outras, ou seja, o local da terapia se tornou além de um espaço para tratar os sofrimentos em um espaço de educação em saúde e participação popular.

Dentre as mudanças após a prática da $\mathrm{TCl}$, podemos citar: adesão da comunidade em participar das terapias comunitárias; o interesse da comunidade em revitalizar a associação comunitária; o aumento do vínculo da comunidade com os profissionais do PSF; o fortalecimento da comunidade que se organizou e escolheu um representante para participar do novo Conselho local de Saúde. Além disso, observou-se o aumento em $100 \%$ do número de prevenções realizadas em nossa unidade; a garantia de acesso a alguns serviços básicos de saúde; e a diminuição da demanda reprimida daquela comunidade que não procurava os serviços de saúde.

A experiência da $\mathrm{TCl}$ na comunidade Alto Jerusalém tem demonstrado adesão pela comunidade e pelos profissionais de saúde, consolidando-se numa estratégia na atenção básica de saúde, além de representar uma ferramenta de integração e consolidação na formação de vínculos e da coletividade social, fomentando ações capazes de transformar a realidade local.

\section{DESAFIOS NA IMPLEMENTAÇÃO DA TERAPIA COMU- NITÁRIA INTEGRATIVA}

Compreendemos que a vida em comunidade é difícil e que os problemas e os conflitos são inevitáveis. Entretanto, eles podem ser fonte de crescimento. Muitas vezes vamos buscar as soluções desses problemas, fora da comunidade, esquecendo que a própria comunidade pode solucionar o problema, sendo capaz de gerir a crise e encontrar uma saída.

Prevenção primária é um conceito comunitário que envolve a diminuição da incidência da doença em uma comunidade pela modificação dos fatores causais, antes que eles tenham oportunidade de causar malefícios ${ }^{(13)}$.

Entendemos que o grande desafio para a promoção e o 
incentivo ao processo de participação comunitária, é saber como envolver as pessoas com seus problemas, suas contradições e adversidades, para agirem juntas, na tentativa de meIhorar o quadro de vida da comunidade.

Ressalta-se que a TCl apresenta-se como uma possibilidade de aproximar os profissionais de saúde, com destaque para o profissional enfermeiro, as demandas da comunidade sendo um instrumento que possibilita uma escuta qualificada, um estar junto, trocar experiências, trabalhar prevenção e promoção da saúde de forma significativa e principalmente, valorizando o saber popular.

\section{CONSIDERAÇÕES FINAIS}

A Terapia Comunitária Integrativa vem se consolidando como uma tecnologia de cuidado, de baixo custo, desenvolvendo ações de promoção da saúde e prevenção do sofrimento emocional nas comunidades, podendo ser considerada, também, uma estratégia de reabilitação e de inclusão social pela rede de apoio psicossocial que ela pode ajudar a construir. Portanto, este estudo vem contribuir, de maneira significativa, para a prática do cuidado.

Como instrumento de cuidado, a TCl demonstrou atender aos princípios norteadores do SUS, ensinando-nos a construir redes de apoio social, possibilitando mudanças sociais e reconhecendo as competências de cada ator social para contribuir na superação das dificuldades.

A Terapia Comunitária Integrativa atende às metas a que se propõe e que deve ser divulgada como uma prática de caráter terapêutico, transformadora da realidade e que pode ser utilizada nos diversos níveis de atenção à saúde, especialmente na atenção básica.

Destacamos ainda a inserção do profissional enfermeiro nesse novo contexto, como terapeuta comunitário. Esperamos que, a partir deste relato, tanto os gestores como as equipes de saúde da família possam incorporar no cotidiano do seu processo de trabalho, a preocupação com o sofrimento emocional da população e terem, na $\mathrm{TCl}$, um referencial de uma tecnologia de cuidado que promova a qualidade de vida das pessoas, que possa gerar processos de mudanças na comunidade, tornando-a mais participativa na conquista de seus direitos e de cidadania.

\section{REFERÊNCIAS}

1. Machado FR, Pinheiro, R, Guizard, FL.Direito a Saúde e a Integralidade no SUS: o Exercício da Cidadania e o Papel do Ministério Público. In: Pinheiro R, Mattos RA, organizadores. Construção Social da Demanda: direito à saúde, trabalho em equipe, participação e espaços públicos. Rio de Janeiro. CEPES/UERJ: ABRASCO, 2005. 304p.

2. Guimarães FJ, Ferreira Filha MO. Repercussões da terapia comunitária no cotidiano de seus participantes. Rev Eletrônica Enferm [periódico na internet]. 2006 [acesso em 12 mar 2010];8(3):404-14 Disponível em: < http://www. fen.ufg.br/revista/revista8_3/v8n3a11.htm. >

3. Ximenes Neto FRG, Costa FAM, Chagas, MIO, Cunha, ICKO. Olhares dos enfermeiros acerca de seu processo de trabalho na prescrição medicamentosa na Estratégia Saúde da Família. Rev Bras Enferm 2007;60(2):133-40.

4. Barreto A. Terapia comunitária passo a passo. Fortaleza: Gráfica LCR; 2005.

5. Ministério da Saúde. Departamento de Atenção Básica. O projeto da terapia comunitária na atenção básica [monografia na internet]. Brasília [acesos em 12 mar 2010]. Disponível em: < http://dtr2004.saude.gov.br/dab/terapia_comunitaria.php $>$

6. Fukui L. Terapia comunitária e o conceito de comunidade: uma contribuição da sociologia [artigo na internet]. São Paulo [acesso em 02 jul 2009]. Disponível em: < http://www.usp.br/nemge/textos_tecendo_estudando/t erapia_comunidade_fukui.pdf02>

7. Ferreira Filha MO, Dias MD, Andrade FB, Lima EAR,
Ribeiro FF, Silva MSS. A terapia comunitária como estratégia de promoção à saúde mental: o caminho para o empoderamento. Rev Eletrônica Enferm [periódico na internet]. 2009 [acesso em 12 mar 2010];11(4): 964-70 Disponível em: < http://www.fen.ufg.br/revista/v11/n4/ v11n4a22.htm >

8. Rocha IA, Braga LAV, Tavares LM, Andrade FB, Ferreira Filha MO, Dias MD, et al. A terapia comunitária como um novo instrumento de cuidado para saúde mental do idoso. Rev Bras Enferm 2009; 62(5):687-94.

9. Bittencourt DL. Na terapia comunitária, a palavra é remédio. COEP. 03 out 2007. Entrevista com Adalberto Barreto [artigo na internet]. [acesso em 12 mar 2010]. Disponível em: < http://www.coepbrasil.org.br/portal/publico/apresentarConteudo.aspx?CODIGO $=$ C200832920140625\& TIPO_ID $=3>$

10. Luisi LV. Terapia comunitária: bases teóricas e resultados práticos de sua aplicação. São Paulo. Dissertação [Mestrado em Psicologia Clínica]- Pontifícia Universidade Católica de São Paulo; 2006.

11. Toro JB, Werneck NMD. Mobilização social: um modo de construir a democracia e a participação. Belo Horizonte: Autêntica; 2004.

12. Wendhausen A. O duplo sentido do controle social: (des) caminhos da participação em saúde. Itajaí (SC): UNIVALI; 2002.

13. Stuart GW, Laraia MT. Enfermagem psiquiátrica. 4. ed. Rio de Janeiro: Reichmann \& Affonso; 2002. 